\title{
Effective magnetic fields induced by EIT in ultra-cold atomic gases
}

\author{
G. Juzeliūnas $\dagger$, J. Ruseckas $\dagger$ and P. Öhberg $\ddagger$ \\ $\dagger$ Institute of Theoretical Physics and Astronomy of Vilnius University, A. Goštauto \\ 12, 01108 Vilnius, Lithuania \\ $\ddagger$ Department of Physics, University of Strathclyde, Glasgow G4 0NG, Scotland \\ E-mail: gj@itpa.It
}

\begin{abstract}
We study the influence of two resonant laser beams (to be referred to as the control and probe beams) on the centre of mass motion of ultra-cold atoms characterised by three energy levels of the $\Lambda$-type. The laser beams being in the Electromagnetically Induced Transparency (EIT) configuration drive the atoms to their dark states. We impose the adiabatic approximation and obtain an effective equation of motion for the dark state atoms. The equation contains a vector potential type interaction as well as an effective trapping potential. We concentrate on the situation where the control and probe beams are co-propagating and have Orbital Angular Momenta (OAM). The effective magnetic field is then oriented along the propagation direction of the control and probe beams. Its spatial profile can be shaped by choosing proper laser beams. We analyse several situations where the effective magnetic field exhibits a radial dependence. In particular we study effective magnetic fields induced by Bessel beams, and demonstrate how to generate a constant effective magnetic field for a ring geometry of the atomic trap. We also discuss a possibility to create an effective field of a magnetic monopole.
\end{abstract}

PACS numbers: 03.75.Ss, 42.50.Gy, 42.50.Fx

Submitted to: J. Phys. B: At. Mol. Opt. Phys. 


\section{Introduction}

Recent experimental advances in trapping and cooling atoms have made it possible to produce atomic Bose-Einstein Condensates (BECs) [1-4] and degenerate Fermi gases [5-7] at temperatures in the microkelvin range. The atomic BECs and degenerate Fermi gases are the systems where an atomic physicist often meets physical phenomena encountered in condensed matter physics. For instance, atoms in optical lattices are often studied using the Hubbard model [8] familiar from solid state physics.

Ultra-cold atomic gases have turned out to be a remarkably good medium for studying a wide range of physical phenomena. This is mainly due to the fact that it is relatively easy to experimentally manipulate parameters of the system, such as the strength of interaction between the atoms, properties of a lattice in which the atoms are trapped, the geometry of an external trap, etc. Such a freedom of manipulating parameters is usually not possible in other systems known from condensed matter or solid state physics.

Atoms forming quantum gases are electrically neutral particles and there is no vector potential type coupling of the atoms with a magnetic field. Therefore, a direct analogy between the magnetic properties of degenerate atomic gases and solid state phenomena is not necessarily straightforward. It is possible to produce an effective magnetic field in a cloud of electrically neutral atoms by rotating the system such that the vector potential will appear in the rotating frame of reference [9-11]. This would correspond to a situation where the atoms feel a uniform magnetic field. Yet stirring an ultracold cloud of atoms in a controlled manner is a rather demanding task.

There have also been suggestions to take advantage of a discrete periodic structure of an optical lattice to introduce assymetric atomic transitions between the lattice sites $[12-15]$. Using this approach one can induce a nonvanishing phase for the atoms moving along a closed path on the lattice, i.e. one can simulate a magnetic flux [12-15]. However such a way of creating the effective magnetic field is inapplicable to an atomic gas that does not constitute a lattice.

A significant experimental advantage would be gained if a more direct way could be used to induce an effective magnetic field. In previous papers [16,17], we have shown how this can be done using two light beams in an Electromagnetically Induced Transparency (EIT) configuration. Here we present a more detailed analysis of the phenomenon for various spatial distributions of the laser fields. We demonstrate that if at least one of these beams contains an Orbital Angular Momentum (OAM), an effective magnetic field appears, which acts on the electrically neutral atoms. In other words, the coupling between the light and the atoms will provide an effective vector potential in the atomic equations of motion. Compared to the rotating atomic gas, where only a constant effective magnetic field is created [9-11], using optical means will be advantageous since the effective magnetic field can now be shaped by choosing proper control and probe beams. The appearance of the effective vector potential is a manifestation of the Berry connection which is encountered in many different areas of physics [18-20]. 
The outline of the paper is as follows. In section 2 we define a system of three level atoms in the $\Lambda$-configuration interacting with the control and probe beams. We allow the two beams to have orbital angular momenta along the propagation axis $z$. In section 3 we present a general treatment of the adiabatic motion of multilevel atoms and apply it to derive the equation of motion for the atom driven to the dark state by the control and probe beams of light. The resulting effective equation of motion contains the effective trapping and vector potentials. In sections 4 and 5 we analyse the effective magnetic field and effective trapping potential in the case where at least one of the laser beams contains an orbital angular momentum. We show that the spatial profile of the effective magnetic field can be controlled by applying proper control and probe beams. We analyse several situations where the effective magnetic field exhibits a radial dependence. In particular we study effective magnetic fields induced by Bessel beams, and demonstrate how to generate a constant effective magnetic field for ring geometry of the atomic trap. We also discuss a possibility to create an effective field of a magnetic monopole. Finally in the concluding section [6 we summarise the findings.

\section{Formulation}

\subsection{The atomic system}

We shall consider an ensemble of atoms characterised by two hyper-fine ground levels 1 and 2, as well as an electronic excited level 3. The atoms interact with two resonant laser beams in the EIT configuration (see figure 1). The first beam (to be referred to as the control beam) drives the transition $|2\rangle \rightarrow|3\rangle$, whereas the second beam (the probe beam) is coupled with the transition $|1\rangle \rightarrow|3\rangle$, as shown in figure 17. The control laser has a frequency $\omega_{c}$, a wave-vector $\boldsymbol{k}_{c}$, and a Rabi frequency $\Omega_{c}$. The probe field, on the other hand, is characterised by a central frequency $\omega_{p}=c k_{p}$, a wave-vector $\boldsymbol{k}_{p}$, and a Rabi frequency $\Omega_{p}$. Of special interest is the case where the probe and control beams can carry OAM along the propagation axis $z$. In that case, the spatial distribution of the beams is given by $[21,22]$

$$
\Omega_{p}=\Omega_{p}^{(0)} \mathrm{e}^{\mathrm{i}\left(k_{p} z+l_{p} \phi\right)}
$$

and

$$
\Omega_{c}=\Omega_{c}^{(0)} \mathrm{e}^{\mathrm{i}\left(k_{c} z+l_{c} \phi\right)},
$$

where $\Omega_{p}^{(0)}$ and $\Omega_{c}^{(0)}$ are slowly varying amplitudes for the probe and control fields, $\hbar \ell_{p}$ and $\hbar \ell_{c}$ are the corresponding orbital angular momenta per photon along the propagation axis $z$, and $\phi$ is the azimuthal angle.

\subsection{Hamiltonian for the electronic degrees of freedom of an atom}

Adopting the rotating wave approximation, the Hamiltonian for the electronic degrees of freedom of an atom interacting with the control and probe fields is in the rotating 
a)

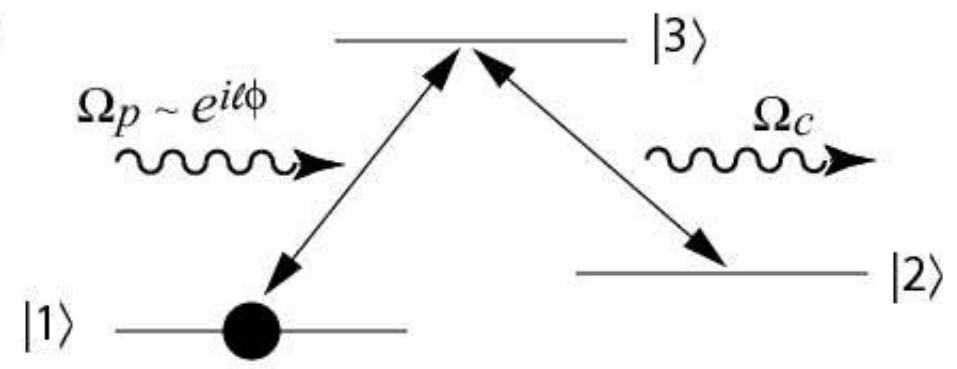

b)

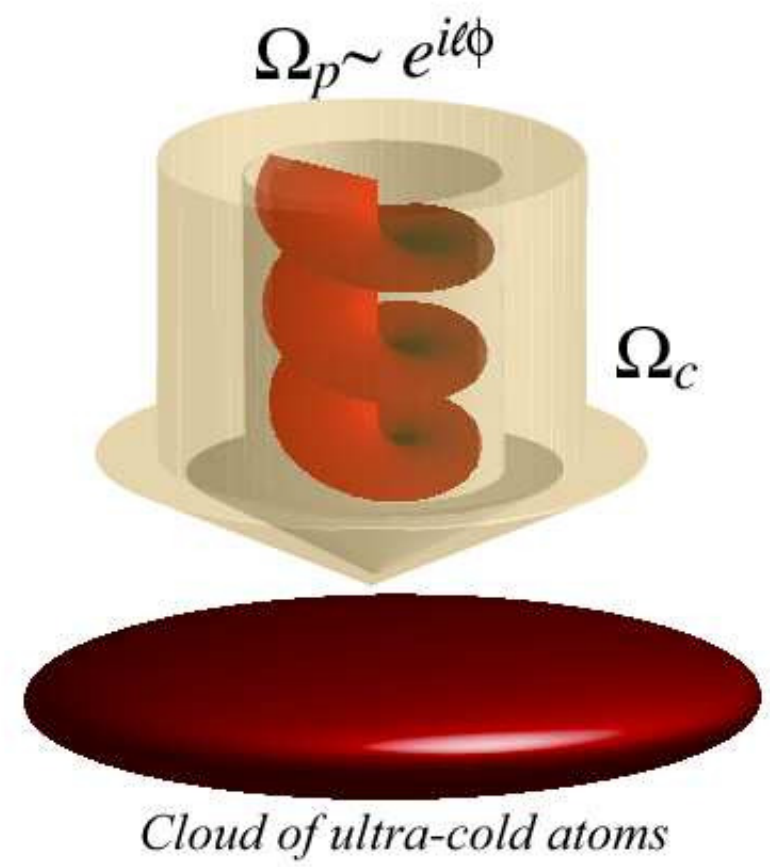

Figure 1. a) The level scheme for the $\Lambda$ type atoms interacting with the resonant probe beam $\Omega_{p}$ and control beam $\Omega_{c}$. b) Schematic representation of the experimental setup with the two light beams incident on the cloud of atoms. The probe field is of the form $\Omega_{p} \sim \mathrm{e}^{\mathrm{i} \ell \phi}$, where each probe photon carry an orbital angular momentum $\hbar \ell$ along the propagation axis $z$.

frame:

$$
\hat{H}_{0}(\boldsymbol{r})=\epsilon_{21}|2\rangle\left\langle 2\left|+\epsilon_{31}\right| 3\right\rangle\langle 3|-\hbar\left(\Omega_{p}|3\rangle\left\langle 1\left|+\Omega_{c}\right| 3\right\rangle\langle 2|+\text { H.c. }\right)
$$

where $\epsilon_{21}=\hbar\left(\omega_{2}-\omega_{1}+\omega_{c}-\omega_{p}\right)$ and $\epsilon_{31}=\hbar\left(\omega_{3}-\omega_{1}-\omega_{p}\right)$ are, respectively, the energies of the detuning from the two- and single-photon resonances, with $\hbar \omega_{j}$ being the electronic energy of the atomic level $j$. Note that the spatial dependence of the Hamiltonian $\hat{H}_{0}(\boldsymbol{r})$ emerges through the spatial dependence of the Rabi frequencies $\Omega_{p} \equiv \Omega_{p}(\boldsymbol{r})$ and $\Omega_{c} \equiv \Omega_{c}(\boldsymbol{r})$.

In what follows the control and probe fields are assumed to be tuned to the twophoton resonance: $\epsilon_{21}=0$. The remaining two photon mismatch (if any) can be 
accommodated within the trapping potential

$$
\hat{V}(\boldsymbol{r})=V_{1}(\boldsymbol{r})|1\rangle\left\langle 1\left|+V_{2}(\boldsymbol{r})\right| 2\right\rangle\left\langle 2\left|+V_{3}(\boldsymbol{r})\right| 3\right\rangle\langle 3| .
$$

where $V_{j}(\boldsymbol{r})$ is the trapping potential for an atom in the electronic state $j$, with $j=1,2,3$. Neglecting the two-photon detuning, the Hamiltonian (3) has the eigenstate

$$
|D\rangle \equiv|D(\boldsymbol{r})\rangle=\frac{1}{\sqrt{1+|\zeta|^{2}}}(|1\rangle-\zeta|2\rangle)
$$

characterised by the zero eigenenergy: $\hat{H}_{0}(\boldsymbol{r})|D\rangle=0$. Here

$$
\zeta=\frac{\Omega_{p}}{\Omega_{c}}
$$

is the ratio of the amplitudes of the probe and control fields.

The state $|D\rangle$ is known as the dark state [23-26]. We shall be interested in a situation where the atoms are driven to their dark states. If an atom is in the dark state $|D\rangle$, the resonant control and probe beams induce the absorption paths $|2\rangle \rightarrow|3\rangle$ and $|1\rangle \rightarrow|3\rangle$ which interfere destructively, resulting in the Electromagnetically Induced Transparency [23-26]. In such a situation, the transitions to the upper atomic level 3 are suppressed, so the atomic level 3 is weakly populated. This justifies neglection of losses due to spontaneous emission by excited atoms in the Hamiltonian (3).

\section{Equations of the atomic motion}

\subsection{Translational motion for a multilevel atom}

Let us now consider translational motion of an atom taking into account its internal degrees of freedom. The full atomic Hamiltonian is given by

$$
\hat{H}=\frac{\hat{p}^{2}}{2 M}+\hat{H}_{0}(\boldsymbol{r})+\hat{V}(\boldsymbol{r})
$$

where $\hat{\boldsymbol{p}} \equiv-\mathrm{i} \hbar \nabla$ is the momentum operator for the atom positioned at $\boldsymbol{r}$, and $M$ is the atomic mass. The Hamiltonian for the electronic degrees of freedom $\hat{H}_{0}(\boldsymbol{r})$ and the external trapping potential $\hat{V}(\boldsymbol{r})$ featured in equation (7) are defined by equations (3) and (41).

For fixed $\boldsymbol{r}$ the electronic Hamiltonian $\hat{H}_{0}(\boldsymbol{r})$ can be diagonalised to give a set of eigenvectors $|X(\boldsymbol{r})\rangle$ and eigenvalues $\varepsilon_{X}(\boldsymbol{r})$, where $X=D,+,-$. The state with $X=D$

is the dark atomic state given by equation (5) and characterised by zero eigenenergy: $\varepsilon_{D}(\boldsymbol{r})=0$. If the single and two photon detuning is zero, the remaining eigenstates are:

$$
| \pm(\boldsymbol{r})\rangle=\frac{1}{\sqrt{2}}(|B\rangle \pm|3\rangle)
$$

corresponding eigenenergies being

$$
\varepsilon_{ \pm}(\boldsymbol{r})= \pm \Omega
$$

Here $\Omega=\sqrt{\Omega_{p}^{2}+\Omega_{c}^{2}}$ is the total Rabi frequency and

$$
|B\rangle \equiv|B(\boldsymbol{r})\rangle=\frac{1}{\sqrt{1+|\zeta|^{2}}}\left(\zeta^{*}|1\rangle+|2\rangle\right)
$$


is the atomic bright state.

The full atomic wave function $\Phi$ can then be expanded as:

$$
|\Phi(\boldsymbol{r})\rangle=\sum_{X=D,+,-} \Psi_{X}(\boldsymbol{r})|X(\boldsymbol{r})\rangle,
$$

where a composite wavefunction $\Psi_{X}(\boldsymbol{r})$ describes the translational motion of an atom in the electronic state $X$, with $X=D,+,-$.

Substituting equation (11) into the Schrödinger equation i $\hbar \partial \Phi / \partial t=\hat{H} \Phi$, one arrives at a set of coupled equations for the components $\Psi_{X}$. Introducing the column $\Psi=\left(\Psi_{D}, \Psi_{+}, \Psi_{-}\right)^{T}$, it is convenient to represent these equations in a matrix form:

$$
\mathrm{i} \hbar \frac{\partial}{\partial t} \Psi=\left[\frac{1}{2 M}(-\mathrm{i} \hbar \nabla-\boldsymbol{A})^{2}+U\right] \Psi,
$$

where $\boldsymbol{A}$ and $U$ are the $3 \times 3$ matrices with the following elements:

$$
\begin{aligned}
& \boldsymbol{A}_{X, X^{\prime}}=\mathrm{i} \hbar\left\langle X \mid \nabla X^{\prime}\right\rangle, \\
& U_{X, X^{\prime}}=\varepsilon_{X}(\boldsymbol{r}) \delta_{X, X^{\prime}}+\left\langle X|\hat{V}(\boldsymbol{r})| X^{\prime}\right\rangle,
\end{aligned}
$$

i.e. the matrix $U$ includes contributions from both the internal atomic energies and also the external trapping potential.

Since the atomic internal motion is much faster than the external (translational)

one, the difference in the atomic energies $U_{X, X}-U_{X^{\prime}, X^{\prime}}$ is normally much larger than the energies of non-adiabatic coupling between these states. In such a situation, the translational motion of atoms in different internal levels can be considered to be independent. This leads to the adiabatic approximation.

\subsection{Effective equation of motion for a dark-state atom}

Specifically, let us suppose that the atomic dark state $|D\rangle$ is well separated from the remaining atomic states $| \pm\rangle$. Neglecting transitions to the latter states, equation (12) provides an effective equation for the translational motion of an atom in the electronic dark state $|D\rangle$ :

$$
\mathrm{i} \hbar \partial \Psi_{D} / \partial t=\hat{H}_{\mathrm{eff}} \Psi_{D}
$$

where the effective Hamiltonian

$$
\hat{H}_{\text {eff }}=\frac{1}{2 M}\left(-\mathrm{i} \hbar \nabla-\boldsymbol{A}_{\text {eff }}\right)^{2}+V_{\text {eff }}
$$

is characterised by the effective vector and trapping potentials:

$$
\boldsymbol{A}_{\text {eff }} \equiv \boldsymbol{A}_{D, D}=\mathrm{i} \hbar\langle D \mid \nabla D\rangle
$$

and

$$
V_{\text {eff }}=U+\phi,
$$


with $U \equiv U_{D, D}$ being defined by the above equation (14). An additional scalar potential $\phi$ appears due to the exclusion of the electronic states with $X= \pm$ in the effective equation of motion (15). In particular, we have

$$
\begin{aligned}
\phi & =\frac{1}{2 M} \sum_{X= \pm} \boldsymbol{A}_{D, X} \boldsymbol{A}_{X, D} \\
& =\frac{\hbar^{2}}{2 M}(\langle\nabla D \mid \nabla D\rangle+\langle D \mid \nabla D\rangle\langle D \mid \nabla D\rangle)
\end{aligned}
$$

and

$$
U=\frac{V_{1}(\boldsymbol{r})+|\zeta|^{2} V_{2}(\boldsymbol{r})}{1+|\zeta|^{2}}
$$

Since $V_{1}(\boldsymbol{r})$ and $V_{2}(\boldsymbol{r})$ are the trapping potentials for an atom in the electronic states 1 and 2 respectively, $U$ represents the external trapping potential for the atom in the dark state.

In this way, the full effective trapping potential $V_{\text {eff }}$ is composed of the external trapping potential $U$ and the geometric scalar potential $\phi$. The former $U$ is determined by the shape of the trapping potentials $V_{1}(\boldsymbol{r})$ and $V_{2}(\boldsymbol{r})$, as well as the intensity ratio $|\zeta|^{2}$. The latter geometric potential $\phi$ is determined exclusively by the spatial dependence of the dark state $|D\rangle$ emerging through the spatial dependence of the ratio between the Rabi frequencies $\zeta=\Omega_{p} / \Omega_{c}$. Note that the effective vector potential $\boldsymbol{A}_{\text {eff }}$ (known as a Berry connection [18]) has a geometric nature as well, because it also originates from the spatial dependence of the atomic dark state $|D\rangle \equiv|D(\boldsymbol{r})\rangle$.

\subsection{Adiabatic condition}

The energy difference between the dark state and the remaining atomic states $| \pm(\boldsymbol{r})\rangle$ is characterised by the total Rabi frequency $\Omega=\sqrt{\Omega_{p}^{2}+\Omega_{c}^{2}}$. Assuming that the control and probe fields are tuned to the one- and two-photon resonances $\left(\epsilon_{31}, \epsilon_{21} \ll \hbar \Omega\right)$, the adiabatic approach holds if the non-diagonal matrix elements in equation (12) are much smaller than the total Rabi frequency $\Omega$. This leads to the following condition

$$
F \ll \Omega
$$

where the velocity-dependent term

$$
F=\frac{1}{1+|\zeta|^{2}}|\nabla \zeta \cdot \boldsymbol{v}|
$$

reflects the two-photon Doppler detuning [17]. Note that the condition (21) does not accommodate effects due to the decay of the excited atoms. The dissipation effects can be included replacing the energy of the one-photon detuning $\epsilon_{31}$ by $\epsilon_{31}-\mathrm{i} \hbar \gamma_{3}$, where $\gamma_{3}$ is the excited-state decay rate. In such a situation, the dark state can be shown to acquire a finite lifetime

$$
\tau_{D} \sim \gamma_{3}^{-1} \Omega^{2} / F^{2}
$$

which should be large compared to other characteristic time scales of the system. 
The condition (21) implies that the inverse Rabi frequency $\Omega^{-1}$ should be smaller than the time an atom travels a characteristic length over which the amplitude or the phase of the ratio $\zeta=\Omega_{p} / \Omega_{c}$ changes considerably. The latter length exceeds the optical wavelength, and the Rabi frequency can be of the order of $10^{7}$ to $10^{8} \mathrm{~s}^{-1}$ [27]. Therefore the adiabatic condition (21) should hold for atomic velocities up to tens of meters per second, i.e. up to extremely large velocities in the context of ultra-cold atomic gases. The allowed atomic velocities become lower if the spontaneous decay of the excited atoms is taken into account. The atomic dark state accquires then a finite lifetime $\tau_{D}$ equal to $\gamma_{3}^{-1}$ times the ratio $\Omega^{2} / F^{2}$, see Eq. (23). The atomic decay rate $\gamma_{3}$ is typically of the order $10^{7} \mathrm{~s}^{-1}$. Therefore if the atomic velocities are of the order of a centimeter per second (a typical speed of sound in an atomic BEC), the atoms should survive in their dark states up to a few seconds. This is comparable to a typical lifetime of an atomic BEC.

\section{Analysis of the effective vector and trapping potentials}

Substituting the expression (51) for the dark state into equation (17) for the effective vector potential, the latter takes the form:

$$
\boldsymbol{A}_{\text {eff }}=\mathrm{i} \hbar \frac{\zeta^{*} \nabla \zeta-\zeta \nabla \zeta^{*}}{2\left(1+|\zeta|^{2}\right)}
$$

The effective magnetic field reads then:

$$
\boldsymbol{B}_{\text {eff }}=\nabla \times \boldsymbol{A}_{\text {eff }}=\mathrm{i} \hbar \frac{\nabla \zeta^{*} \times \nabla \zeta}{\left(1+|\zeta|^{2}\right)^{2}}
$$

and the geometric scalar potential is

$$
\phi=\frac{\hbar^{2}}{2 M} \frac{\nabla \zeta^{*} \nabla \zeta}{\left(1+|\zeta|^{2}\right)^{2}} .
$$

\subsection{Separation into the amplitude and phase}

Let us express the ratio of Rabi frequencies $\zeta$ in terms of the amplitude and phase:

$$
\zeta=\frac{\Omega_{p}}{\Omega_{c}}=|\zeta| \mathrm{e}^{\mathrm{i} S} .
$$

The effective vector potential, the effective magnetic field, and the effective scalar potential then read

$$
\begin{aligned}
& \boldsymbol{A}_{\mathrm{eff}}=-\hbar \frac{|\zeta|^{2}}{1+|\zeta|^{2}} \nabla S, \\
& \boldsymbol{B}_{\mathrm{eff}}=\hbar \frac{\nabla S \times \nabla|\zeta|^{2}}{\left(1+|\zeta|^{2}\right)^{2}} \\
& \phi=\frac{\hbar^{2}}{2 M} \frac{(\nabla|\zeta|)^{2}+|\zeta|^{2}(\nabla S)^{2}}{\left(1+|\zeta|^{2}\right)^{2}} .
\end{aligned}
$$




\subsection{Representation in terms of the mixing angle}

It is convenient to introduce the mixing angle $\alpha$ via the following relationships:

$$
\sin \alpha=\frac{1}{\sqrt{1+|\zeta|^{2}}}, \quad \cos \alpha=\frac{|\zeta|}{\sqrt{1+|\zeta|^{2}}} .
$$

If the intensity ratio $|\zeta|^{2}$ is much larger than the unity, the mixing angle is $\alpha \approx 1 / \zeta$. On the other hand, if $|\zeta|^{2} \ll 1$, we have $\alpha \approx \pi / 2-|\zeta|$.

The dark state can now be represented as

$$
|D\rangle=\sin \alpha|1\rangle-\cos \alpha \mathrm{e}^{\mathrm{i} S}|2\rangle .
$$

The effective vector and scalar potentials can also be rewritten in terms of the mixing angle:

$$
\boldsymbol{A}_{\mathrm{eff}}=-\hbar \cos ^{2} \alpha \nabla S=-\frac{\hbar}{2}(1+\cos (2 \alpha)) \nabla S
$$

and

$$
\begin{aligned}
\phi & =\frac{\hbar^{2}}{2 M}\left[\left(\frac{1}{2} \sin (2 \alpha) \nabla S\right)^{2}+(\nabla \alpha)^{2}\right] \\
& =\frac{\hbar^{2}}{8 M}\left[\left(1-\cos ^{2}(2 \alpha)\right)(\nabla S)^{2}+\frac{(\nabla \cos (2 \alpha))^{2}}{1-\cos ^{2}(2 \alpha)}\right],
\end{aligned}
$$

i.e. both potentials can be expressed through the quantity

$$
\cos (2 \alpha)=\frac{|\zeta|^{2}-1}{|\zeta|^{2}+1} .
$$

The same applies to the effective magnetic field:

$$
\boldsymbol{B}_{\mathrm{eff}}=\nabla \times \boldsymbol{A}_{\mathrm{eff}}=\frac{\hbar}{2} \nabla S \times \nabla \cos (2 \alpha)
$$

\subsection{Co-propagating control and probe beams with $O A M$}

If the co-propagating probe and control fields carry OAM, their amplitudes $\Omega_{p}$ and $\Omega_{c}$ are given by equations (11)-(2). The phase of the ratio $\zeta=\Omega_{p} / \Omega_{c}$ then reads

$$
S=l \phi,
$$

where $l=l_{p}-l_{c}$. Note that although both the control and probe fields are generally allowed to have non-zero OAM by equations (11)-(2), it is desirable for the OAM to be zero for one of these beams. In fact, if both $l_{p}$ and $l_{c}$ were non-zero, the amplitudes $\Omega_{p}$ and $\Omega_{c}$ should simultaneously go to zero along the $z$-axis. In such a situation, the

total Rabi frequency $\Omega=\sqrt{\Omega_{p}^{2}+\Omega_{c}^{2}}$ would also vanish, leading to the violation of the adiabatic condition (21) along the $z$-axis.

Substituting equation (37) into equations (33), (34) and (36), one has

$$
\begin{aligned}
& \boldsymbol{A}_{\mathrm{eff}}=-\hbar \cos ^{2} \alpha \frac{l}{\rho} \boldsymbol{e}_{\varphi}, \\
& \phi=\frac{\hbar^{2}}{2 M}\left[\left(\frac{1}{2} \sin (2 \alpha) \frac{l}{\rho}\right)^{2}+(\nabla \alpha)^{2}\right],
\end{aligned}
$$


and

$$
\boldsymbol{B}_{\text {eff }}=\frac{\hbar}{2} \frac{l}{\rho} \boldsymbol{e}_{\varphi} \times \nabla \cos (2 \alpha)
$$

where $\boldsymbol{e}_{\varphi}$ is the unit vector in the azimuthal direction, and $\rho$ is the cylindrical radius.

In what follows the intensity ratio $|\zeta|^{2}$ is considered to depend on the cylindrical radius $\rho$ only (unless stated otherwise). In that case the effective scalar potential and magnetic field reduce to

$$
\phi=\frac{\hbar^{2}}{2 M}\left[\left(\frac{1}{2} \sin (2 \alpha) \frac{l}{\rho}\right)^{2}+\left(\frac{\partial \alpha}{\partial \rho}\right)^{2}\right]
$$

and

$$
\boldsymbol{B}_{\mathrm{eff}}=-\frac{\hbar}{2} \frac{l}{\rho} \frac{\partial}{\partial \rho} \cos (2 \alpha) \boldsymbol{e}_{z}
$$

In such a situation the effective magnetic field is directed along the $z$-axis.

\section{Specific cases}

\subsection{Polynomial case}

If we take

$$
|\zeta|=a \rho+b \rho^{2}
$$

then

$$
\cos (2 \alpha)=\frac{\left(a \rho+b \rho^{2}\right)^{2}-1}{\left(a \rho+b \rho^{2}\right)^{2}+1} .
$$

Consequently one has:

$$
\begin{aligned}
& \boldsymbol{A}_{\mathrm{eff}}=-\hbar l \frac{\rho(a+b \rho)^{2}}{1+\rho^{2}(a+b \rho)^{2}} \boldsymbol{e}_{\varphi}, \\
& \phi=\frac{\hbar^{2}}{2 M} \frac{\left(l^{2}+1\right) a^{2}+2\left(l^{2}+2\right) a b \rho+\left(l^{2}+4\right) b^{2} \rho^{2}}{\left(1+\rho^{2}(a+b \rho)^{2}\right)^{2}} .
\end{aligned}
$$

In this case the effective magnetic field

$$
\boldsymbol{B}_{\mathrm{eff}}=-\hbar l \frac{2(a+b \rho)(a+2 b \rho)}{\left(1+\rho^{2}(a+b \rho)^{2}\right)^{2}} \boldsymbol{e}_{z}
$$

exhibits a radial dependence.

\subsection{Bessel beam}

Suppose the probe field represents a Bessel beam and the Rabi frequency of the control beam is almost constant within an atomic cloud. In such a case we have

$$
\zeta=b J_{l}(a \rho) \mathrm{e}^{\mathrm{i} l \varphi}
$$


where $b$ is a dimensionless constant determining the relative strength of the probe field. The effective vector and scalar potentials are then:

$$
\begin{aligned}
& \boldsymbol{A}_{\mathrm{eff}}=-\hbar \frac{b^{2} J_{l}(a \rho)^{2}}{1+b^{2} J_{l}(a \rho)^{2}} \frac{l}{\rho} \boldsymbol{e}_{\varphi}, \\
& \phi=\frac{\hbar^{2} b^{2}}{2 M} \frac{4 l^{2} J_{l}(a \rho)^{2}+a^{2} \rho^{2}\left(J_{l-1}(a \rho)-J_{l+1}(a \rho)\right)^{2}}{4 \rho^{2}\left(1+b^{2} J_{l}(a \rho)^{2}\right)^{2}} .
\end{aligned}
$$

In this case the effective magnetic field

$$
\boldsymbol{B}_{\text {eff }}=-\hbar \frac{a b^{2} l}{\rho} \frac{J_{l}(a \rho)\left(J_{l-1}(a \rho)-J_{l+1}(a \rho)\right)}{\left(1+b^{2} J_{l}(a \rho)^{2}\right)^{2}} \boldsymbol{e}_{z}
$$

also exhibits a radial dependence. Furthermore, the strength of the effective magnetic field alternates its sign, i.e. the regions with the effective magnetic field aligned along $z$ axis are replaced by the regions in which the effective magnetic field is directed opposite to the $z$-axis and vice versa.

Next we shall examine situations where the effective magnetic field is constant.

\subsection{Constant effective magnetic field for ring geometry}

In the previous paper [17] we have analysed a constant effective field in the case where the atomic motion is restricted to distances where $\rho<\rho_{\max }$. This can be achieved if the intensity ratio is:

$$
|\zeta|^{2}=\frac{\left(\rho / \rho_{\max }\right)^{2}}{1-\left(\rho / \rho_{\max }\right)^{2}}
$$

so that the effective vector potential, equation (38), takes the form

$$
\boldsymbol{A}_{\text {eff }}=-\hbar l \rho \rho_{\max }^{-2} \boldsymbol{e}_{\phi} .
$$

This yields a constant effective magnetic field

$$
\boldsymbol{B}_{\mathrm{eff}}=-2 \hbar l \rho_{\max }^{-2} \boldsymbol{e}_{z},
$$

with the corresponding cyclotron frequency $\omega_{c}=\hbar 2 l / M \rho_{\max }^{2}$ and the magnetic length $\ell_{B}=\sqrt{\hbar / M \omega_{c}}=\rho_{\max } / \sqrt{2 l}$.

Let us now consider a situation where the atomic motion is restricted additionally from below, i.e. $\rho>\rho_{\min }$. In such a case the constant effective magnetic field is obtained provided

$$
|\zeta|^{2}=\frac{\rho^{2}-\rho_{\min }^{2}}{\rho_{\max }^{2}-\rho^{2}} .
$$

The effective vector potential then takes the form

$$
\boldsymbol{A}_{\text {eff }}=-\hbar \frac{\rho^{2}-\rho_{\min }^{2}}{\rho_{\max }^{2}-\rho_{\min }^{2}} \frac{l}{\rho} \boldsymbol{e}_{\varphi},
$$

giving the following magnetic field strength:

$$
\boldsymbol{B}_{\mathrm{eff}}=-\frac{2 \hbar l}{\rho_{\max }^{2}-\rho_{\min }^{2}} \boldsymbol{e}_{z} .
$$




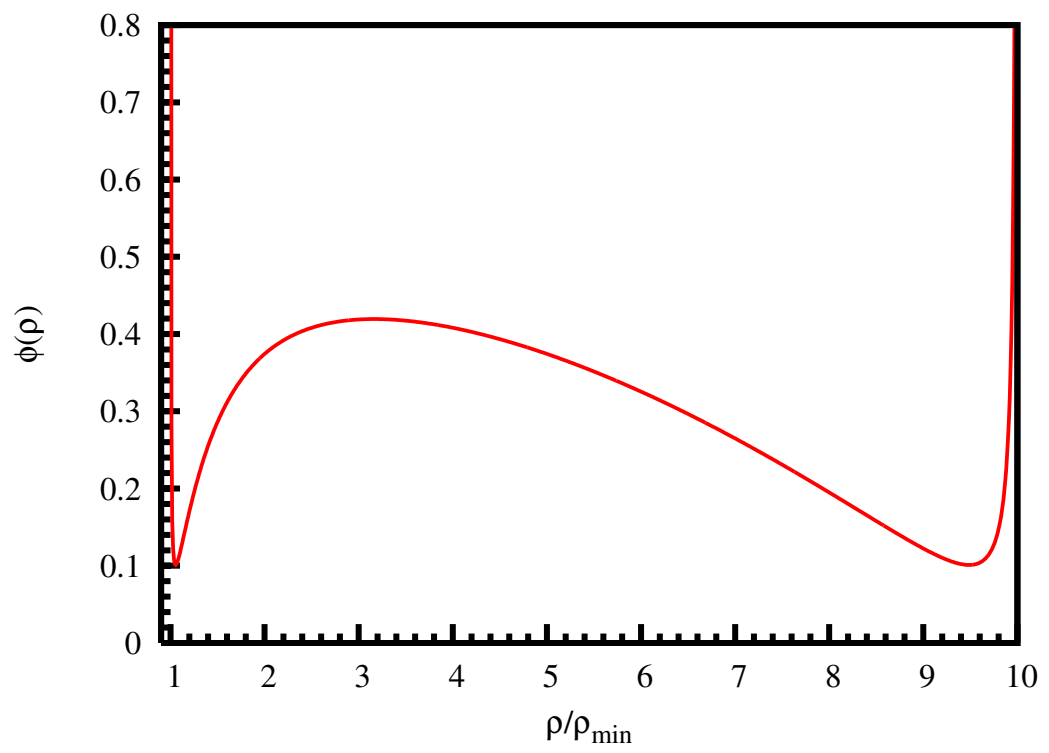

Figure 2. Geometric scalar potential $\phi$ for the case of constant effective magnetic field $B_{\text {eff }}$ in the ring geometry. The constants are $M=1, \hbar=1, l=10, \rho_{\min }=1$, and $\rho_{\max }=10$.

For $\rho \rightarrow \rho_{\min }$ and $\rho \rightarrow \rho_{\max }$, the intensity ratio $|\zeta|^{2}$ goes respectively to zero and infinity, so the equations (54)-(56) have a meaning only within a disc where $\rho_{\min }<\rho<\rho_{\max }$. In other words, equation (54) can model an actual intensity distribution of the control and probe beams only within this region. The effective magnetic flux over the disc is given by $\Phi=2 \pi \hbar l$. Since the winding number $l$ of light beams can currently be as large as several hundreds $[28,29]$, it is possible to induce a substantial flux $\Phi$ in the atomic cloud. This might enable us to study phenomena related to filled Landau levels with a large number of atoms in the quantum gas.

Finally the scalar potential is given by

$$
\phi=\frac{\hbar^{2}}{2 M}\left(\frac{l^{2}}{\rho^{2}} \frac{\left(\rho_{\max }^{2}-\rho^{2}\right)\left(\rho^{2}-\rho_{\min }^{2}\right)}{\left(\rho_{\max }^{2}-\rho_{\min }^{2}\right)^{2}}+\frac{\rho^{2}}{\left(\rho_{\max }^{2}-\rho^{2}\right)\left(\rho^{2}-\rho_{\min }^{2}\right)}\right) .
$$

The potential $\phi$ has singuliarities both at $\rho=\rho_{\min }$ and $\rho=\rho_{\max }$, as illustrated in figure 2. This might provide a natural trapping container confining the atoms within the ring.

\subsection{Effective magnetic field of a magnetic monopole}

The method for creating an effective magnetic field allows us to consider various exotic cases. An interesting possibility is to create an effective field of a magnetic monopole. Such an idea was proposed in [30].

A possible expression for the vector potential of the monopole field has the form

$$
\boldsymbol{A} \sim \frac{1-\cos \theta}{r \sin \theta} \boldsymbol{e}_{\varphi}
$$


where $r, \theta$ and $\varphi$ are the spherical coordinates. Such a potential has a singularity at the string where $\theta=0$. Unlike in [30] we do not attempt to eliminate this singularity, since the elimination does not make the field esier to create.

In order to get an effective vector potential of a magnetic monopole the intensity ratio should obey the condition

$$
|\zeta|^{2}=\frac{1-\cos \theta}{1+\cos \theta}
$$

where we are no longer making the paraxial approximation. In such a situation

$$
\cos (2 \alpha)=-\cos \theta=-z / r .
$$

Consequently the effective vector potential, the effective magnetic field and the scalar potential are

$$
\begin{aligned}
& \boldsymbol{A}_{\text {eff }}=-\frac{\hbar l}{2} \frac{1-\cos \theta}{r \sin \theta} \boldsymbol{e}_{\varphi}, \\
& \boldsymbol{B}_{\text {eff }}=-\frac{\hbar l}{2 r^{2}} \boldsymbol{e}_{r}, \\
& \phi=\frac{\hbar^{2}}{2 M} \frac{l^{2}+1}{4 r^{2}} .
\end{aligned}
$$

The magnetic charge of the effective monopole is now proportional to the difference of the orbital angular momentum of the light beams $\hbar l \equiv \hbar\left(l_{p}-l_{c}\right)$. On the other hand, the emerging scalar potential $\phi$ is repulsive and spherically symmetrical, and is characterised by the $r^{-2}$ behaviour.

In order to satisfy the condition (58) the Rabi frequencies should obey the following:

$$
\begin{aligned}
\left|\Omega_{p}\right|^{2} & =f(\boldsymbol{r})(1-\cos \theta), \\
\left|\Omega_{c}\right|^{2} & =f(\boldsymbol{r})(1+\cos \theta)
\end{aligned}
$$

where $f(\boldsymbol{r})$ is an arbitrary function of the coordinates. For a light beam with an OAM, its intensity is known to be zero along the propagation axis $z[21,22]$. Therefore if the probe beam has an OAM, the function $f(\boldsymbol{r})$ should be zero for $\cos \theta=-1$, i.e. along the negative part of $z$-axis. Under this condition the control beam should also be zero along the negative part of $z$-axis, so the adiabatic condition (21) is violated there. Similar conclusions can be reached if the control beam has OAM.

In this way the effective field of a magnetic monopole cannot be created in the whole space, i.e. the effective field differs from the field of a monopole in the vicinity of the negative (or positive) part of the $z$-axis. This conclusion is valid even if the singularity of the potential is eliminated [30], since the intensities of the beams remain of the same form using such a procedure. It should be noted that a posibilitiy of creating the field of a magnetic monopole can be improved applying a more complex scheme where three laser beams act resonantly on four-level atoms in the tripod configuration [31].

\section{Conclusions}

In this paper we have studied the effects of using probe and control beams with orbital angular momentum in an EIT configuration. The nontrivial phase and intensity of 
the incident light beams gives rise to effective magnetic vector potentials and trapping potentials for the atoms. The theory holds for both fermions and bosons where the effects from collisional interactions can be readily included [17]. Recent advances in spatial light modulator technology enables us to consider rather exotic light beams [32]. Indeed one of the advantages of using light to create the effective vector potential, and consequently an effective magnetic field, is the freedom to choose almost any spatially dependent effective magnetic field, as long as the corresponding light fields obey Maxwell's equations. This means we have complete freedom to choose the effective magnetic field in a two-dimensional geometry, but we can also control the effective field in three dimensions. Shaping light beams in three dimensions is more difficult but certainly not impossible [33]. We have analysed different cases where the radial dependence of the magnetic fields was exploited. In particular the homogenous magnetic field in a ring geometry and magnetic fields using Bessel beams were studied.

An effective magnetic field acting on an atomic quantum gas offers some truly remarkable possibilities. We are now in a position to study magnetic effects encountered in solid state situations with electrons. The effective magnetic field can also be applied to investigate other intriguing phenomena which intrinsically depend on the magnetic field. For instance, the properties of a gas described by a single completely filled Landau level can now be explored using a cold gas of electrically neutral atomic fermions [34]. In addition, if the collisional interaction between the atoms is taken into account, we can study the magnetic properties of a superfluid atomic Fermi gas [35-38] where insight into the BCS-BEC crossover regime could be gained.

\section{Acknowledgments}

This work was supported by the Royal Society of Edinburgh, the Alexander von Humboldt Foundation and the Marie-Curie Trainings-site at the University of Kaiserslautern. Helpful discussions with M. Fleischhauer are gratefully acknowledged.

\section{References}

[1] Davis K B, Mewes M-O, Andrews M R, van Druten N J, Durfee D S, Kurn D M and Ketterle W 1995 Phys. Rev. Lett. 753969

[2] Bradley C C, Sackett C A, Tollett J J and Hulet R G 1995 Phys. Rev. Lett. 751687

[3] Dalfovo F, Giorgini S, Pitaevskii L and Stringari S 1999 Rev. Mod. Phys. 71463

[4] Pitaevskii L and Stringari S 2003 Bose-Einstein Condensation (Oxford: Clarendon Press)

[5] DeMarco B and Jin D 1999 Science 2851703

[6] Schreck F, Khaykovich L, Corwin K L, Ferrari G, Bourdel T, Cubizolles J and Salomon, C 2001 Phys. Rev. Lett. 87080403

[7] Hadzibabic Z, Gupta S, Stan C A, Schunck C H, Zwierlein M W, Dieckmann K and Ketterle W 2003 Phys. Rev. Lett. 911604012005 Phys. Rev. Lett. 95010404

[8] Jaksch D, Bruder C, Cirac J I, Gardiner C W and Zoller, P 1998 Phys. Rev. Lett. 813108

[9] Bretin V, Stock S, Seurin Y and Dalibard J 2004 Phys. Rev. Lett. 92050403

[10] Schweikhard V, Coddington I, Engels P, Mogendorff V P and Cornell, E A 2004 Phys. Rev. Lett. 92040404 
[11] Baranov M A, Osterloh K and Lewenstein M 2005 Phys. Rev. Lett. 94070404

[12] Jaksch D and Zoller P 2003 New J. Phys. 556

[13] Mueller E J 2004 Phys. Rev. A 70 041603(R)

[14] Sørensen A S, Demler E and Lukin M D 2005 Phys. Rev. Lett. 94086803

[15] Osterloh K, Baig M, Santos L, Zoller P and Lewenstein M 2005 Phys. Rev. Lett. 95010403

[16] Juzeliūnas G and Öhberg P 2004 Phys. Rev. Lett. 93033602

[17] Juzeliūnas G, Öhberg P, Ruseckas J and Klein A 2005 Phys. Rev. A 71053614

[18] Jackiw R 1988 Comments At. Mol. Phys. 2171

[19] Sun C-P and Ge M-L 1990 Phys. Rev. D 411349

[20] Dum R and Olshanii M 1996 Phys. Rev. Lett. 761788

[21] Allen L, Padgett M and Babiker M 1999 Prog. Opt. 39291

[22] Allen L, Barnett S M and Padgett M J 2003 Optical Angular Momentum (Bristol: Institute of Physics)

[23] Arimondo E 1996 Progr. Opt. 35259

[24] Harris S E 1997 Phys. Today 5036

[25] Matsko A B, Kocharovskaja O, Rostovtsev Y, Welch G R, Zibrov A S and Scully M O 2001 Advances in Atomic, Molecular, and Optical Physics 46191

[26] Lukin M D 2003 Rev. Mod. Phys. 75457

[27] Hau L V, Harris S E, Dutton Z and Behrooz C 1999 Nature 397594

[28] Courtial J, Dholakia K, Allen L and Padgett M J 1997 Opt. Commun. 144210

[29] Curtis J E, Koss B A and Grier D G 2002 Opt. Commun. 207169

[30] Zhang P, Li Y and Sun C P 2005 Eur. Phys. J. D 36229

[31] Ruseckas J, Juzeliūnas G, Öhberg P and Fleischhauer M 2005 Phys. Rev. Lett. 95010404

[32] McGloin D, Spalding G, Melville H, Sibbett W and Dholakia K 2003 Opt. Express 11158

[33] Whyte G and Courtial J 2005 New J. Phys 7117

[34] Öhberg P, Juzeliūnas G, Ruseckas J and Fleischhauer M Phys. Rev. A at press

[35] Zwierlein M W, Stan C A, Schunk C, Raupach S M F, Gupta S, Hadzibabic Z and Ketterle W 2003 Phys. Rev. Lett. 91250401

[36] Regal C A, Greiner M and Jin D S 2004 Phys. Rev. Lett. 92040403

[37] Bourdel T, Khaykovich L, Cubizolles J, Zhang J, Chevy F, Teichmann M, Tarruell L, Kokkelmans S J J M F and Salomon C 2004 Phys. Rev. Lett. 93050401

[38] Zwierlein M W, Abo-Shaeer J R, Schirotzek A, Schunck C H and Ketterle W 2005 Nature 435 1047 